\title{
Total Quality Management in the Hospital Area and Its Contribution to Patient Safety
}

\author{
Marcia Galdino Pereira \\ marciagpereira@gmail.com \\ Angelo Maia Cister \\ cister@facc.ufrj.br
}

\begin{abstract}
Objective: This study deals with the emergence of Total Quality Management and its start in the hospital area, as well as its connection to the patient safety issue, introducing the use of quality tools as the "cause and effect diagram", brainstorming, flowcharts for analysis of adverse events and a financial analysis of the impact that adverse events cause in an institution. Methods: We used a literature review through consultation with research sources as Scielo, Medline, Fiocruz - Proqualis, as well as access to books and websites on the subject. Conclusion: With the increasing need to provide hospital care quality, Total Quality Management in the hospital area has much to contribute to reach an even better service and safe, helping to mitigate the adverse events through use of tools such as "cause and effect diagram", brainstorming and flowcharts, where the occurrence of events involving patient safety resulted in large losses of hospital costs.
\end{abstract}

Keywords: total quality management, quality, safety, adverse event.

\section{Introduction}

It is common to observe constant advances in Medicine in the health area, either through the creation of new drug formulas, new methods of treatment or new technologies, causing information and knowledge to become essential for those working in the field in order to develop their technical skills, but also for the institutions in order to create strategies for a greater and improved performance providing better patient care in an increasingly competitive market.

It is common to find a major concern of health institutions with the quality of customer service against market instability, greater competitiveness and a growing level of consumer demand.Offering a quality service does notmean providing the service in the best manner. It is essential to meet customer needs, be patient or familiar, as well as to perform tasks with a focus on productivity, and for that matter, three elements become essential: people, equipment and procedures. The methodology of Total Quality Management is inserted in this very context, consisting of a management approach that seeks to create internal conditions ensuring the survival of organizations in the long term.

The Total Quality Management (TQM) consists of a methodology of identifying the real needs of customers in order to ensure the preference thereof, that is, for them to continue with the need and desire of the product/service. For that matter, the participation of everyone in the organization becomes essential forreaching such result so that the provision of funds and knowledge can promote increased productivity. In addition, total 
quality control aims at creating and maintaining standards that meet customer needs, as well as the continuous improvement of such standards from a strategic view of the business.

The health quality concern dates far back. In 1854, the introduction of the first continuous improvement model of quality in healthcare occurred, provided by British nurse Florence Nightingale; her work during the Crimean War (after six months working in Scutari Hospital)resulted in a 40\% reduction in mortality rate. Such result was achieved by establishing strict sanitary and nursing care standards set by her, namely, reduction in the mortality rate occurred from the moment hospital attention was directed to the observation of risks involving patients and poor hygiene in hospitals.

Ensuring health care quality is assuring all care for the patient will be done safely. However,according to data from the 2000 United States Institute of Medicine, mistakes associated with health care cause between 44,000 and 98,000 dysfunctions each year in US hospitals, which also occurs in Europe; such data show that one in ten patients in European hospitals suffer avoidable damage and adverse events caused during care received.

Given the above, this paper aims to present a theoretical review of Total Quality Management and its introduction in the hospital area of how the use of management tools - "cause and effect diagram", brainstorming and flowcharts can assist in risk management towards greater safety in patient care, and finally, how the occurrence of adverse events impact the financial results of the institution.

\section{Methodology}

This article seeks to present points for a greater understanding of the subject from an exploratory stage of research bibliographical studies in order to prepare material that corroborates to a greater understanding and to create new ideas regarding the introduction of the total quality management methodology in hospital care, and how its use will assist in a safer practice at a time characterized by the concern of institutions with patient safety and reducing costs.

The theoretical concept is based on a qualitative research approach, so data were collected at national and international levels through consultation with research sources, such as Scielo, Medline, Fiocruz - Proqualis, as well as access to books, websites and journals on the subject.The keywords were: total quality management, quality, safety, adverse event.

\section{Development}

\section{History of Total Quality Management: industry $x$ health}

The beginning of work focused on quality occurred in the 20's with W.A. Shewhart, a North American statistician who developed a Process Statistical Control (PSC) system and the PDCA (Plan, Do, Check and Action) Cycle, whose tool became known years later.Laterin the 50s and 60s, American authors such as W. Edwards Deming, Joseph M.Juran and Armand V. Feigenbaum developed the TQM - Total Quality Management concept, in order to integrate development, maintenance and improvement efforts.

In the 80s, after World War II, Deming, Juran and Crosby founded JUSE (Japanese Union of Scientists and Engineers). Deming conducted lectures to train entrepreneurs and industrialists on statistical process control and quality management at a time Japan was rebuilding itself, which resulted in the improvement of this management practice with the main objective of increasing productivity, thereby raising the interest of heads of large companies. This practice is known in Japan as TQC (Total Quality Control) to differentiate the model previously created in the 50 's by Feigenbaum. 
The Total Quality Management model was initially used in industries, and involved the participation of everyone in the institution, including top management,which supported this methodology. That was the key for disseminating concepts for the working class party, which ended up regularly using the tools of quality proposed by Deming, Juran and Ishikawa, consolidating the idea of total quality control. Deming andJuran reinforced the participation of the senior leadership in the performanceand involvement in the process, and the need for constancy of the purpose of leaders, since quality management not only should be part of the strategy, but it should also be present in various processes, causing improvement to be observed and practiced in different ways and in several sectors of an organization. According to Juran, the quality-related triad consists of planning, control and improvement.

Even though the dimensions of Total Quality (Quality, Cost, Delivery, Morale and Safety) are fully applicable to a hospital organization where a number of issues must be eliminated, aiming at cost reduction, reduction in the average length of stay, and the fact that all of the above should be accomplished ensuring safe patient care, yet, the concepts related to Total Quality Management were opposed to in the hospital area of countries with healthcare systems that advocated universal reach; in turn, they were accepted in places focused on competition and cost reduction, such as the United States.

Globalization and the political and financial scenario associated with the growth of user requirements, as well as highest demands and desire of health insurance companies (regarding private units) for greater control gave rise to the beginning of the application of Total Quality Management in Brazilian health organizations in the late twentieth century, all of which to ensure positive results and customer satisfaction in a constantly evolving market that increasingly requires an improved performance. As to the health sector, it is also noteworthy that besides the concern with the welfare issue in health institutions, it is necessary to emphasize other extremely important points for an organization, such as: need for planning, forward thinking, human resources (especially regarding selection and training), information and communication system (both for internal employees and stakeholders), safety, adequacy of the physical structure, and monitoring and control of hospital infections - items that will influence the final outcome of the service.

The first entity to stimulate Brazilian hospitals in developing quality programs was the Paulista Medical Association (APM), which started the AIJ (Medical and Hospital Care Quality Program), in São Paulo, based on the model brought to Brazil in the 1990s by the National Quality Foundation. This model consists of tasks performed by managers, whether they are in a private entity or otherwise.

\section{Risk management: beginning and development}

The search for Total Quality also involves offering a product/service with minimum risk possible, so that the Risk Management process refers to any situation that may generate an unexpected result. Risk management began in the Unites States, in the 50's, in large US companies and it focused onthe purchase of their insurance and management policies. In the health area, management began a few years later due to the growth and high complexity of health facilities, whether hospitals, diagnostic centers, blood banks, among others, whose main objective was to protect healthcare organizations against losses by taking out insurance to cover actions/proceedings against professionals/hospitals.

However, a specific Hospital Risk Management program began in the US only after the "malpractice insurance crisis" that was characterized, among several reasons, for the increase in the number of claims for negligence. Such methodology aimed at an improvement in the quality of care, patient and professional safety, 
as well as in minimizing avoidable expenditure. Later, hospital risk management was implemented in other countries in Europe, Oceania and Latin America.

Given what was mentioned above, errors and damages related to patients during hospital care were already much-discussed facts. In 2000, data were disclosed in the United States indicating that errors related to health care account for approximately 44 a 98 thousand adverse events/per year in hospitals, which triggered a global movement focused on patient safety, as more people die from medical errors than from aviation errors, breast cancer and AIDS.

In Brazil, Hospital Risk Management only began in 2001 through the National Health Surveillance Agency (Anvisa) and the Ministry of Health with project Sentinela Hospitals. Nevertheless, reviews of quality in health strengthened the hospital safety matter, since the implementation of an accreditation program seeks to ensure quality in every hospital service, whose main focus will be hospital processes.

According to the World Health Organization, patient safety was defined as "the reduction of risk of unnecessary damage associated with health care to an acceptable minimum", i.e., such acceptableminimum refers to what is feasible given current knowledge, available resources and the context in which service was held against the risk of not treating or against other treatment. It is estimated that in developed countries, one in ten patients receiving hospital care suffer damage or injury resulting from such care, which can range from severe to fatal, and more than half of the adverse events could have been avoided.

Considering hospital medical assistance as one of the most complex and dynamic provided by humans and that culminate with the occurrence of numerous errors that could compromise patient safety, we emphasize the importance of focusing on the analysis of the system as a whole to seek improvements in hospital care; such fact was observed in high-risk sectors, such as industry and aviation, whose major purpose is to develop human error prevention systems, instead of focusing on people, thus creating or stimulating a culture of punishment of the individual in the institution.

The occurrence of an error/event demonstrates the presence of failure(s) in the process involving several factors; such fact is explained by the Swiss cheese theory by James Reason (Figure 1), where for an event/damage to occur to a patient, there should be several "gaps", ranging from structural failures, malpractice and risk behavior also by patients. However, such failures should be investigated in order tobe corrected and prevented, including the definition of actions to be taken upon the impossibility to prevent their occurrence.

As previously mentioned, the problem that caused the error is multifactorial and its cause may be related to the structure, process and outcome, as well as equipment and materials, procedures, and the human being. Such points should be thoroughly analyzed in order to identify the real and main causes that led to the error.

In order to analyze the factors that caused the error and seek the root cause, tool "cause and effect diagram," also known as "fishbone diagram" and "ishikawa diagram"(Figure 2) can be used. This tool helps detecting several possible causes that may have generated the undesirable effect (event/damage). It is common to associate the brainstorming technique to the "cause and effect diagram" for all possible causes to be considered.

The cause and effect diagram will assist in the analysis of several points: Method (analysis of how the event was being carried out); Material (analysis of which materials were being used in the procedure, for example); Labor (analysis of the professional's skills, what may have influenced him/her, whether there was malpractice or not, etc.); Machine (analysis of all matters as to the operation of the machine); Measure (analysis 
of cases involving measurement, calibration tools, etc.); Environment (factor analysis involving the environment - space, temperature, etc.). Therefore, the identification of the causes will occur through analyzing the points in the diagram.

It is noteworthy that mistakes can be correlated with Clinical Management and Administrative Management. Once the Administrative Management provides subsidies to Clinical Management through several areas: maintenance, purchasing, etc.,it is essential, upon the investigation and analysis of incidents, for an action plan to be developed in orderto enable a safer working environment that will reflect in improved care. For example: development of institutional protocols focused on best practices that will specify which appropriate preventive measures should be taken to minimize the risk to the patient.

In this context a Hospital Risk Management Committee should be formed, with an investigative and technical-scientific character in order to develop policies, protocols and standardsin the areas of risk prevention involving care and management processes, and to encourage/improve risk management of all sectors of the organization. A strong safety culture in the institution will assist the reduction of adverse events through learning from mistakes or in a proactive manner (acting preventively), also through a redesign of processes hence, the importance of using flowcharts, i.e., a sequential illustration of all process steps.

The flowcharts are one of the quality tools aimed at identifying improvement opportunities, identifying critical points that can lead to errors favoring the establishment of prevention barriers, and improving communication between people. Ingeneral, the flowcharts will promote a better understanding of the process itself, which will favor the determination of main items of control, and will assist in process management. Moreover, they will help to achieve quality assurance and increased productivity.

It is worth mentioning the importance of risk management as it relates to hospital costs, since the events, whether severe or fatal, will create consequences that can be increasingly costly for both families and for healthcare systems. That will reflect both financially and in hospital production, as regards, for example, the increase of the average length of stay. Thus, health care undergoes unnecessary inflations reflecting higher costs for the patient due to errors involving care, i.e., if care were carried out appropriately, the cost would not be the same.

Preventable adverse events, e.g., related to drugs, can cost hospitals about two billion dollars in the United States, and such fact was also observed in countries such as Ireland and the UK, where the costs related to length of stay in hospitals generated by adverse events generate about two billion pounds in cost, and the expense of the National System of Health regarding legal issues associated with adverse events is 400 million pounds/year. It is also noteworthy that much is spent with the need of repeating diagnostic tests, and such fact will affect the financial department of the institution, along with errors resulting in the loss of confidence and the reduction of satisfaction of patients and health professionals.

\section{Conclusion}

The fact is, if the main purpose is providing high quality medical and hospital care, it is necessary to spread the concepts of the Total Quality Management methodology throughout the organization, of how quality tools can provide subsidies and information for decision-making, and also, how they will assist in the understanding of the hospital process holistically, so that this methodology can contribute to a better understanding of how areas interact with each other and how they can contribute to the achievement of better results. 
Greater engagement between the administrative and clinical management (healthcare) is also essential, and the balance in the performance of these managements will be reflected in a higher quality of care. Therefore, process mapping, establishment of protective barriers and subsequent improvement thereof is also extremely important, and such fact will also ensure a safer care, since safety will be evident from the interaction of processes.

In addition, patient safety must always be present in the organization's strategy, aiming at a constant strengthening of the safety culture that will reflect in an increasing number of notifications - which will assist in identifying critical areas and factors, but also in stimulating open discussions of errors increasingly eliminating punishment culture from the institution.

In conclusion, a total quality program in health institutions will improveboth financial and assistance gains, nevertheless, an improved analysis of the costs arising from adverse events generated in Brazilian institutions and their impact on the final outcome of the institution are also necessary. It is important to emphasize that safety is the starting point for improvingthe quality of care and positive results for everyone involved. 